\title{
Detection of copy number variants and loss of heterozygosity from impure tumor samples using whole exome sequencing data
}

\author{
XIAOCHENG LIU ${ }^{1}$, AO LI $^{1,2}$, JIANING XI $^{1}$, HUANQING FENG ${ }^{1}$ and MINGHUI WANG ${ }^{1,2}$ \\ ${ }^{1}$ Department of Electronic Science and Technology, School of Information Science and Technology, University \\ of Science and Technology of China; ${ }^{2}$ Center for Biomedical Engineering, School of Information Science \\ and Technology, University of Science and Technology of China, Hefei, Anhui 230027, P.R. China
}

Received January 16, 2016; Accepted June 2, 2017

DOI: $10.3892 / \mathrm{ol} .2018 .9150$

\begin{abstract}
Using whole-exome sequencing (WES) for the detection of chromosomal aberrations from tumor samples has become increasingly popular, as it is cost-effective and time efficient. However, factors which present in WES tumor samples, including diversity in exon size, batch effect and tumor impurity, can complicate the identification of somatic mutation in each region of the exon. To address these issues, the authors of the present study have developed a novel method, PECNV, for the detection of genomic copy number variants and loss of heterozygosity in WES datasets. PECNV combines normalized logarithm ratio of read counts (Log Ratio) and B allele frequency (BAF), and then employs expectation maximization (EM) algorithm to estimate parameters involved in the models. A comprehensive assessment of PECNV of PECNV was performed by analyzing simulated datasets contaminated with different normal cell proportion and eight real primary triple-negative breast cancer samples. PECNV demonstrated superior results compared with ExomeCNV and EXCAVATOR for the detection of genomic aberrations in WES data.
\end{abstract}

Correspondence to: Dr Ao Li, Department of Electronic Science and Technology, School of Information Science and Technology, University of Science and Technology of China, 443 Huang Shan Road, Shu Shan, Hefei, Anhui 230027, P.R. China

E-mail: aoli@ustc.edu.cn

Abbreviations: WES, whole exome sequence; BAF, B allele frequency; EM, expectation maximization; $\mathrm{CNV}$, copy number variants; LOH, loss of heterozygosity; array-CGH, microarray comparative genomic hybridization; SNP, single nucleotide polymorphism; NGS, next-generation sequencing; WGS, whole-genome sequencing; PCA, principal component analysis; HMM, hidden Markov model; TNBC, primary triple-negative breast cancer

Key words: copy number variant, expectation maximization algorithm, exome sequencing, loss of heterozygosity

\section{Introduction}

Somatic aberrations, including copy number variants (CNV) and loss of heterozygosity ( $\mathrm{LOH})$ have an important role in tumor progression (1-3). Previous experimental studies of somatic aberrations in tumor samples were performed primarily by using microarray comparative genomic hybridization (array-CGH) techniques $(4,5)$ or single nucleotide polymorphism (SNP) arrays (6,7). More recently next-generation sequencing (NGS) was developed, which allows for massively parallel sequencing of DNA (7-9). The NGS technology platform is able to efficiently sequence a sample in a few days, which is much shorter compared with previous methods (10). Whole-exome sequencing (WES) employs NGS technology and only sequences the exonic regions, and dismisses the intragenic regions.

Compared with whole-genome sequencing (WGS), WES has the advantages of more straightforward interpretation, lower cost and significantly greater coverage, which contributes to improvements in quality of data (11-13).

Despite the promising potential of WES for detecting $\mathrm{CNV}$ from tumor samples, several critical issues are required to be addressed. When using WES data issues, including batch effect (14) among samples and the sparse nature of exonic regions, make algorithms that use split-read or read-pair signals unsuitable for CNV detection (15). To address these issues, several computational methods have been proposed to identify CNV from exome sequencing samples, including ExomeCNV (16), Copy Number Inference From Exome Reads (CoNIFER) (17), XHMM (18), CANOES (19) and EXCAVATOR (20). CoNIFER uses singular value decomposition to correct data, while XHMM employs principal component analysis to eliminate noise included in raw read depth signal and builds a hidden Markov model to discover CNVs in each exonic region $(17,18)$. XHMM calculates the breakpoint quality score, which contributes to further downstream analysis. Although CoNIFER and XHMM have been reported to have good performance, many samples need to be provided at once, which limit their application to situations where there is a limited availability of sequencing samples. CANOES overcomes this limitation and uses a regression-based approach to estimate parameters in a negative binomial model (19). However, all of these methods have been specially developed to detect rare 
CNVs, while common CNVs also carry substantial risk for disease (20). Furthermore, these methods classify each exonic region into one of three state classifications: Deletion, normal or amplification, which cannot provide the exact copy number of each exon $(19,20)$. EXCAVATOR has been introduced to classify genomic regions into 5 copy number states using a hidden Markov model. However, this method cannot infer tumor impurity in tumor samples and identify $\mathrm{LOH}$, which is common in cancer genome (21).

To overcome the limitations of existing CNV detection methods using WES data, a novel method, PECNV, is presented in the current study to identify CNVs and $\mathrm{LOH}$ from tumor and matched normal samples using WES data. Comprehensive processing procedures, including elimination of sequencing/mapping bias, batch effect and exon size diversity, were used in PECNV to normalize read counts derived from tumor WGS data. PECNV combined log ratio of read counts (Log Ratio) and B allele frequency (BAF) with two Gaussian models, which take tumor impurity and Log Ratio baseline shift into account. Expectation maximization algorithm was applied to estimate parameters included in the models. Copy number and $\mathrm{LOH}$ in each exonic region were estimated using the optimal parameters. A comprehensive assessment of PECNV was performed by analyzing simulated samples contaminated with different proportions of normal cells and eight real primary triple-negative breast cancer WES sequencing datasets. PECNV showed superior results compared with ExomeCNV and EXCAVATOR in genomic aberrations detection using tumor WES data.

\section{Materials and methods}

Data biases and correction. To study genomic CNVs from exome sequencing data, read counts (RC) aligned to each exon and BAF signals were obtained from the tumor sequencing file using SAMtools, as previously described (22). RC for each exonic region displays the number of reads aligned to each region, which can reflect copy numbers in exonic regions. As previously reported, there are primarily four sources of bias that affect RC signals, including the size of exonic regions, batch effect, local GC content percentage and genomic mappability $(14,23)$. To eliminate the effect of different exon sizes among regions and make the data among samples comparable, reads counts per thousand bases per million reads sequenced (RPKM) were calculated as introduced by Mortazavi et al (24):

$$
R P K M=\frac{10^{9} \cdot R C}{T R C \cdot S}
$$

where $T R C$ refers to total read counts mapped to exonic regions, and $s$ is the size of the captured exonic region. Next, normalization methods as described in Yoon et al (23) were employed to remove GC content and mappability bias. Following the procedure, GC-content and mappability were scaled to integer values. Then, the normalized RPKM was calculated using the following formula:

$$
N R P K M=R P K M \times \frac{m}{m_{x}}
$$

where $N R P K M$ is the corrected $R P K M, m$ is the median of RPKM of all exonic regions, and $\mathrm{m}_{\mathrm{x}}$ refers to the median
$R P K M$ of the regions, which share the same GC-content and mappability as the current exonic region. Following GC and mappability correction, the logarithmic ratio of RPKM (Log Ratio) between tumor samples and matched normal samples in each corresponding region was calculated to eliminate batch effect.

Statistical distributions of the signals. Previous studies $(16,21)$ have shown that both Log Ratio and BAF approximately follow a normal distribution. Accordingly, let $r_{i}$ be Log Ratio and $c_{i}$ be copy number states, as defined in (21) of the $i$-th exon in the genomic sequence. A total of four global parameters were employed in the PECNV model: Tumor impurity $(w), \log$ Ratio baseline shift $(o)$, and standard deviation of Log Ratio $\left(\sigma_{x}\right)$ and BAF signals $\left(\sigma_{b}\right)$. Then, the conditional probability density function follows a normal distribution:

$$
r \mid w, o, \sigma_{r}, \mathrm{c} \sim N\left(\log \left(\frac{y_{c}}{2}\right)+o, \sigma_{r}^{2}\right)
$$

where $y_{c}$ refers to the average copy number in state $c$, which is defined as:

$$
y_{c}=w \cdot n_{s}+(1-w) \cdot n_{c}
$$

where $n_{s}$ denotes the normal copy number $\left(n_{s}=2\right)$, and $n_{c}$ is the tumor copy number in state $c$.

Similarly, BAF can be modeled by a normal distribution as reported in (21). Let $b_{i}$ be the BAF signal in $i$-th exonic region, and its conditional probability density function can be formulated as:

$$
b \mid w, \sigma_{b}, c \sim N\left(\mathrm{~B}_{w, c}, \sigma_{b}^{2}\right)
$$

where $B_{w, c}$ denotes theoretical BAF in mixed tumor cells and can be calculated as:

$$
B_{w, \mathrm{c}}=\frac{w+(1-\mathrm{w}) \cdot b_{c}}{2 \cdot w+(1-\mathrm{w}) \cdot n_{c}}
$$

where $b_{c}$ refers to theoretical BAF in the state in pure tumor samples.

EM algorithm and CBS segmentation. The EM algorithm was used to estimate the global parameters. EM is a general method, which identifies the optimal parameters that maximize the logarithm likelihood function when an incomplete data set is given (25). Parameters that hold same value in all exons in one given sample may be termed global parameter. Specifically, given signals ( $\log$ Ratio or BAF) $X=\left\{x_{1}, x_{2} \ldots\right.$ ,$\left.x_{N}\right\}$, copy number states $C=\left\{c_{1}, c_{2} \ldots, c_{N}\right\}$ and global parameter set $\Theta=\left\{\sigma_{r}, \sigma_{b}, 0, w\right\}$, the likelihood can be formulated as:

$$
\begin{aligned}
& P(\mathrm{X}, \mathrm{C} \mid \Theta)=\prod_{i=1}^{N} p\left(\mathrm{x}_{i}, \mathrm{c}_{i} \mid, \Theta\right) \\
& =\prod_{i=1}^{N} p\left(\mathrm{x}_{i} \mid \mathrm{c}_{i}, \Theta\right) p\left(\mathrm{c}_{i} \mid \Theta\right)
\end{aligned}
$$

where $p\left(c_{i} \mid \Theta\right)$ can be treated as constant, because it is assumed that copy number states follow a uniform distribution, and copy number states are independent of the set parameter. Given the observation sequence and conditional probability 
density function, the authors of the present study aimed to estimate parameters that maximize the likelihood so that it best matches the observations in order to elucidate the corresponding state sequence. In PECNV, the EM algorithm was implemented to identify the optimal parameters. As the first step, the EM algorithm calculated the expected value of the $\log$-likelihood $\log (P(X, C \mid \Theta)$ given the signal $\mathrm{X}$ and the current estimated parameters (E step), in which the expectation of log likelihood can be formed as:

$$
\begin{aligned}
& \left.Q\left(\Theta, \Theta^{(\mathrm{i}-1)}\right)=\mathrm{E}\left[\log \mathrm{p}(\mathrm{X}, \mathrm{C} \mid \Theta) \mid \mathrm{X}, \Theta^{(\mathrm{i}-1)}\right]\right) \\
& =\sum_{i=1}^{N} \sum_{c_{i}=1}^{M} \log p\left(\mathrm{x}_{i}, \mathrm{c}_{i} \mid \Theta\right) \cdot \mathrm{f}\left(\mathrm{c}_{i} \mid \mathrm{X}, \Theta^{(\mathrm{i}-\mathrm{l})}\right)
\end{aligned}
$$

where $M$ is the total number of copy number states, $\Theta^{(\mathrm{i}-1)}$ refers to the parameter estimated in (i-1)-th iteration, and $\Theta$ is the next iteration. $f\left(c_{i} \mid X, \Theta^{(i-1)}\right)$ is the posterior probability given both signal $\mathrm{X}$ and current estimated parameter $\Theta^{(\mathrm{i}-1)}$. Note that in this equation, $\mathrm{X} \Theta^{(\mathrm{i}-1)}$ and are constants, and $\Theta$ is a normal variable that we wish to adjust. Then, the Log Ratio and BAF expectation can be denoted as $Q_{\mathrm{r}}\left(\Theta, \Theta^{(\mathrm{i}-1)}\right)$ and $Q_{\mathrm{b}}\left(\Theta, \Theta^{(\mathrm{i}-1)}\right)$, respectively.

As both CNV and LOH can span multiple exons, the PECNV method was used to call $\mathrm{CNV} / \mathrm{LOH}$ on larger segments, in which exons are contiguous in the human genome. Both Log Ratio and BAF were segmented by the CBS algorithm in order to identify the somatic mutation boundaries as described previously (26). Following segmentation, all exons within the same segment share the same CNV state. Following segmentation, posterior probability $f\left(c_{i} \mid X, \Theta^{(\mathrm{i}-1)}\right)$ was calculated in each exon. As the probability depends on the signals that are in the same segment, $f\left(c_{i} \mid X, \Theta^{(\mathrm{i}-1)}\right)$ can be formulated as:

$$
\begin{aligned}
& f\left(\mathrm{c}_{i} \mid \mathrm{X}, \Theta^{(\mathrm{i}-1)}\right)=p\left(\mathrm{c}_{i} \mid \mathrm{x}_{j 1}, \mathrm{x}_{j 2}, \ldots \mathrm{x}_{j m}, \Theta^{(\mathrm{i}-1)}\right) \\
& =\prod_{k=1}^{m} p\left(\mathrm{c}_{i} \mid \mathrm{x}_{j k}, \Theta^{(\mathrm{i}-1)}\right) \\
& =\prod_{k=1}^{m} p\left(\mathrm{x}_{j k} \mid \mathrm{c}_{i}, \Theta^{(\mathrm{i}-1)}\right) \cdot \mathrm{p}\left(\mathrm{c}_{i} \mid \Theta^{(\mathrm{i}-1)}\right) / \mathrm{p}\left(\mathrm{x}_{j k} \mid \Theta^{(\mathrm{i}-1)}\right)
\end{aligned}
$$

where $x_{\mathrm{j} 1}, x_{\mathrm{j} 2}, \ldots x_{j m}$ are signals in the $j$-th segment that the $i$-th exonic region belongs to, and $m$ is the number of exons in that segment. $p\left(x_{j k} \mid \Theta^{(\mathrm{i}-1)}\right)$ can be calculated as:

$$
\begin{aligned}
& p\left(\mathrm{x}_{j k} \mid \Theta^{(\mathrm{i}-1)}\right)=\sum_{c=1}^{M} p\left(\mathrm{x}_{j k}, \mathrm{c} \mid \Theta^{(\mathrm{i}-1)}\right) \\
& =\sum_{c=1}^{M} p\left(\mathrm{c} \mid \Theta^{(\mathrm{i}-1)}\right) \rtimes \mathrm{p}\left(\mathrm{x}_{j k} \mid \mathrm{c}, \Theta^{(\mathrm{i}-1)}\right)
\end{aligned}
$$

The M-step of the EM algorithm is to find $\Theta$ to maximize the expectation that was computed in the E step:

$$
\Theta^{(i)}=\arg \max _{\Theta}\left(Q_{r}\left(\Theta, \Theta^{(i-1)}\right)+Q_{b}\left(\Theta, \Theta^{(i-1)}\right)\right)
$$

where $\Theta^{(i)}$ is adopted for next iteration in the E-step. It is difficult to obtain in closed-form expression by directly solving the equation (10). Instead in the M step, the Newton algorithm (27) was used to identify optimal parameters. Steps E and M were repeated until the algorithm converged, and then parameters in the last iteration were returned as the optimal estimators.
Using optimal parameters, CNV states that the product of posterior probability of Log Ratio, and BAF was chosen as the final state in that exonic region.

Statistical analysis. Average absolute difference is employed to measure the difference between different methods results and the ground truth. Supposing there exists two signals $S_{1}=\left\{s_{11}, s_{12}, s_{13} \ldots s_{1 M}\right\}$ and $S_{1}=\left\{s_{21}, s_{22}, s_{23} \ldots s_{2 M}\right\}$, which share the same dimension of $\mathbf{M}$. The average absolute difference (denoted as AAD) between signals and can be calculated as:

$$
\operatorname{AAD}\left(\mathrm{S}_{1}, \mathrm{~S}_{2}\right)=\frac{1}{M} * \sum_{i=1}^{M} \mid s_{1 i}-s_{2 i}
$$

The smaller the value of average absolute difference, the closer the two signals are.

Simulated and real WES data of tumor samples. To evaluate the performance of PECNV in different tumor impurity samples, 8 tumor samples dataset were simulated with paired normal at $\mathrm{x} 100$ coverage where normal cell proportion ranged from 0.1 to 0.8 . The simulation method was proposed by CLImAT (28), which employed tumor-normal admixture on chromosome 20 of the human reference genome. For better simulation of real situations, the test genome was constructed according to the CNV state obtained from a real WES sample. To generate a simulated dataset, reads were sampled from both control and test genomes with different tumor impurities and different coverage to be mapped to the reference genome. For each simulated sample, a total of 72,389 exons were generated with 29,134 amplified exons and 43,255 deleted exons. With these simulated samples, a comprehensive evaluation of PECNV for CNV detection was performed. For real sequencing data, 8 paired primary triple-negative breast cancer (TNBC) WES samples were randomly selected from datasets employed by Shah et al (29) and were used in the present study. The reads were previously sequenced on the Illumina Genome Analyzer IIx platform and mapped to the reference genome NCBI36/hg18 using Burrows-Wheeler Aligner (22). The data were downloaded from the European Genome-Phenome Archive (https://www.ebi.ac.uk/ega/home; accession no. EGAS00001000132).

Availability of PECNV. The PECNV software package implemented by Matlab is freely available from: https://github. com/lxcheng/PECNV. Using a Windows 7 operating system with $2.6 \mathrm{GHz}$ CPU and $4 \mathrm{G} \mathrm{RAM}$, it takes $\sim 10 \mathrm{~min}$ to process a single tumor sample. RC and BAF can be obtained from the tumor sequencing file using SAMtools (22).

\section{Results}

Estimation of tumor impurity of simulated datasets. The simulated samples were detected by PECNV, and the results are shown in Fig. 1A. PECNV was able to accurately predict tumor impurity between 0.1-0.8 (sum of square difference to ground truth equals to $1.83 \times 10^{-4}$ ), indicating PECNV can efficiently estimate the proportion of tumor cells from samples with different levels of tumor impurities. The accurate 
estimation of tumor impurity indicates a good performance in detecting CNVs.

Next, the latest version of EXCAVATOR (version 2.2) was compared with PECNV using the simulated datasets Copy number accuracy of the results was calculated and shown in Fig. 1B. The accuracy of EXCAVATOR decreased as tumor impurity increased, particularly when tumor impurity was between 0.3-0.5. When tumor impurity was $>0.6$, the detection accuracy was maintained at $\sim 0.6$. By contrast, PECNV achieved a high accuracy $(>0.99)$ in all 8 simulated samples, suggesting that PECNV is able to accurately predict copy number in samples with different levels of tumor impurity.

Comparison of $C N V$ detection of simulated datasets using different methods. Sensitivity and specificity values were calculated to compare the performance of PECNV and EXCAVATOR in the detection of CNVs in simulated samples at 100x coverage. The results are shown in Fig. 2. When tumor impurity was $<0.3$, good results were obtained with PECNV and EXCAVATOR, with average sensitivity and specificity values $>0.98$. However, as the tumor impurity increased $>0.40$, EXCAVATOR showed a reduction in power to detect CNV as sensitivity started to decrease. However, a high specificity was maintained ( $>0.99$ ), which indicated that EXCAVATOR is relatively conservative in identifying CNVs. Compared with EXCAVATOR, PECNV exhibited strong robustness to tumor impurity and obtained high sensitivity and specificity across all simulated samples. These results indicated that PECNV is able to accurately identify CNVs even when tumor impurity is relatively high.

Samples with 200x and 300x coverage were also simulated to evaluate the performance of PECNV (Fig. 3) in deep sequenced samples. Overall, PECNV achieved a good performance in terms of sensitivity and specificity in the simulated tumor samples with tumor impurity at three different sequencing coverage. As tumor impurity varies in tumor samples with different coverage, the performance of PECNV remains excellent, demonstrating the robustness of PECNV to tumor impurity. Furthermore, there is an improvement in sensitivity at 200x and 300x coverage with PECNV compared with $100 \mathrm{x}$, when tumor impurity is 0.9 . This finding suggested that PECNV has the potential to identify CNVs in highly contaminated tumor samples, which have been deep sequenced.

Estimation of tumor impurity of TNBC datasets. PECNV, EXCAVATOR and ExomeCNV were applied to 8 real paired TNBC WES samples. To assess the performance of PECNV, the corresponding SNP-arrays assayed by Affymetrix SNP6.0 array (Affymetrix; Thermo Fisher Scientific, Inc., Waltham, MA, USA) were detected by Allele-Specific Copy number Analysis of Tumors (ASCAT) (30), and these results were used as the ground truth. As the first step, tumor impurity values estimated by ASCAT and PECNV were assessed (Table I). The tumor impurity values detected by ASCAT ranged from 0.28 to 0.59 . The estimated tumor impurity of WES data detected by PECNV was consistent with the results attained by ASCAT (average of absolute difference, $4.8 \times 10^{-2}$ ).

Next, the average copy number (ACN) of the tumor samples as detected by the different methods was assessed
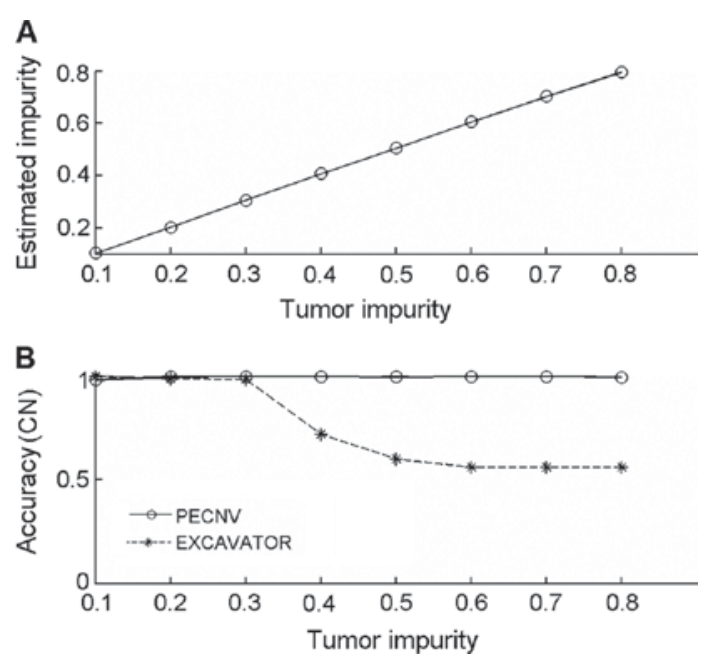

Figure 1. Comparison of PECNV and EXCAVATOR. (A) Tumor impurity estimated by PECNV. (B) Accuracy of copy number detection. CN, copy number.
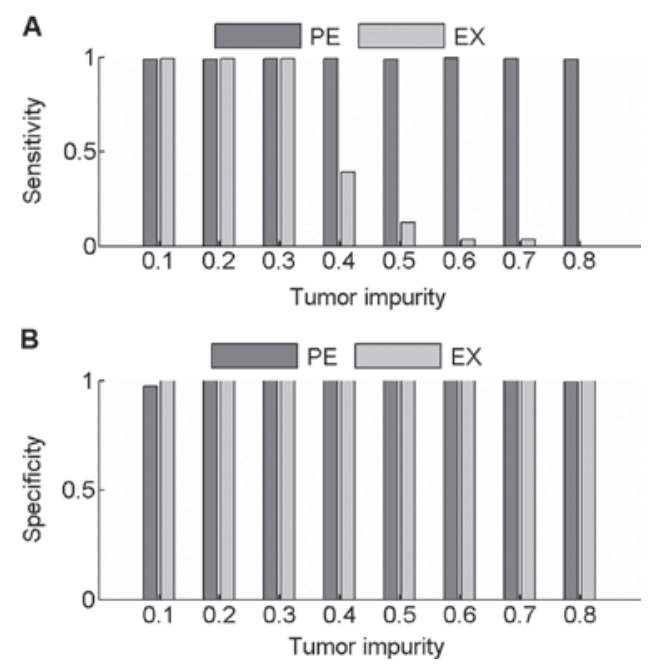

Figure 2. Performance of PECNV and EXCAVATOR for detection of CNVs in simulated samples with different tumor impurities. Comparisons of (A) sensitivity and (B) specificity. PE, PECNV; EX, EXCAVATOR.
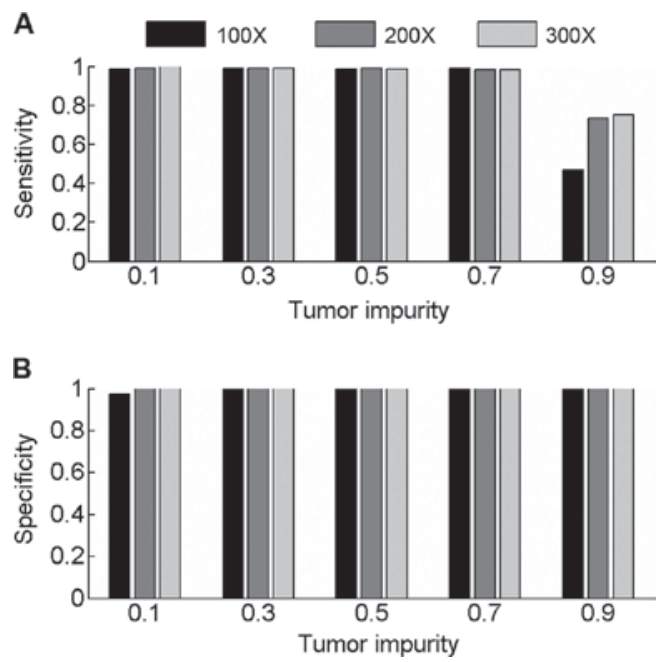

Figure 3. Performance of the PECNV method for detection of copy number variants in simulated samples with different tumor impurities at 100x, 200x and 300x coverage. Comparisons of (A) sensitivity and (B) specificity. 
Table I. ACN and tumor impurity estimated by ASCAT, PECNV, EXCAVATOR and ExomeCNV in 8 real primary triple-negative breast cancer datasets.

\begin{tabular}{|c|c|c|c|c|c|c|}
\hline \multirow[b]{2}{*}{ Sample } & \multicolumn{2}{|c|}{ ASCAT } & \multicolumn{2}{|c|}{ PECNV } & \multirow{2}{*}{$\begin{array}{c}\text { EXCAVATOR } \\
\text { ACN }\end{array}$} & \multirow{2}{*}{$\begin{array}{c}\text { ExomeCNV } \\
\text { ACN }\end{array}$} \\
\hline & $\mathrm{ACN}$ & Impurity & $\mathrm{ACN}$ & Impurity & & \\
\hline $\mathrm{S} 1$ & 3.62 & 0.37 & 3.60 & 0.40 & 2.26 & 2.08 \\
\hline $\mathrm{S} 2$ & 1.55 & 0.52 & 1.67 & 0.57 & 2.00 & 2.06 \\
\hline $\mathrm{S} 3$ & 1.85 & 0.59 & 2.06 & 0.64 & 2.03 & 2.02 \\
\hline $\mathrm{S} 4$ & 2.56 & 0.56 & 2.76 & 0.60 & 2.00 & 2.11 \\
\hline S5 & 1.74 & 0.49 & 2.06 & 0.61 & 2.18 & 2.00 \\
\hline S6 & 2.54 & 0.33 & 2.60 & 0.30 & 2.07 & 2.01 \\
\hline S7 & 2.30 & 0.58 & 2.43 & 0.59 & 2.11 & 2.04 \\
\hline S8 & 1.87 & 0.28 & 1.92 & 0.33 & 2.16 & 1.87 \\
\hline
\end{tabular}

ACN, average copy number.

Table II. Total number of copy number gains, losses and exons in 8 real primary triple-negative breast cancer datasets.

\begin{tabular}{lrrrr}
\hline Sample & \multicolumn{1}{c}{ Gains } & Losses & \multicolumn{1}{c}{ Total } & Exons \\
\hline S1 & 125,779 & 2,610 & 128,389 & 164,318 \\
S2 & 2,864 & 70,686 & 73,550 & 164,318 \\
S3 & 39,350 & 44,650 & 84,000 & 164,318 \\
S4 & 64,993 & 776 & 65,769 & 164,318 \\
S5 & 21,278 & 68,809 & 90,087 & 164,318 \\
S6 & 71,267 & 9,884 & 81,151 & 164,318 \\
S7 & 63,587 & 31,385 & 94,972 & 164,318 \\
S8 & 24,397 & 49,899 & 73,996 & 164,318 \\
\hline
\end{tabular}

Exons, the total number of exons.

Table III. Loss of heterozygosity, sensitivity, specificity and accuracy of PECNV and ExomeCNV.

\begin{tabular}{llllllll}
\hline & \multicolumn{3}{c}{ PECNV } & & \multicolumn{3}{c}{ ExomeCNV } \\
\cline { 2 - 4 } \cline { 6 - 7 } Sample & SEN & SPE & ACC & & SEN & SPE & ACC \\
\hline S1 & 0.94 & 0.99 & 0.98 & & 0.99 & 0.05 & 0.25 \\
S2 & 0.80 & 1.00 & 0.91 & & 0.98 & 0.09 & 0.47 \\
S3 & 0.99 & 0.99 & 0.99 & & 0.99 & 0.12 & 0.84 \\
S4 & 1.00 & 0.98 & 0.99 & & 1.00 & 0.21 & 0.73 \\
S5 & 0.80 & 0.98 & 0.90 & & 0.77 & 0.69 & 0.72 \\
S6 & 0.99 & 0.99 & 0.99 & & 0.99 & 0.09 & 0.72 \\
S7 & 0.92 & 0.98 & 0.95 & & 0.99 & 0.06 & 0.63 \\
S8 & 0.97 & 0.99 & 0.99 & 0.99 & 0.18 & 0.50 \\
\hline
\end{tabular}

SEN, sensitivity; SPE, specificity; ACC, accuracy.

(Table I). EXCAVATOR provided a good estimate of ACN in tumor samples where ACN was close to 2 (such as S3 and S7).
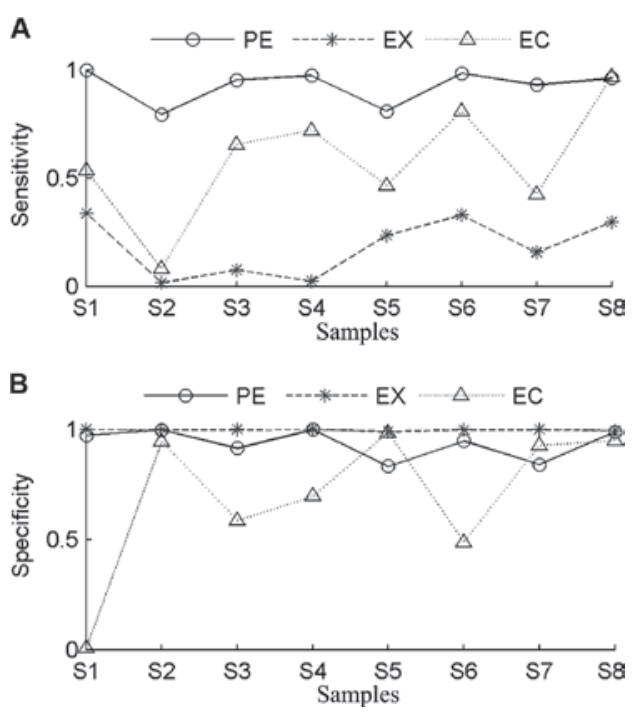

Figure 4. Sensitivity and specificity of PECNV, EXCAVATOR and ExomeCNV for detection of copy number variants in primary triple-negative breast cancer datasets. Comparisons of (A) sensitivity and (B) specificity. PE, PECNV; EX, EXCAVATOR; EC, ExomeCNV.

ExomeCNV achieved reasonable results in tumor samples with ACN lower than 2, particularly for tumor sample S8 where the ACN was 1.87. By comparison, the ACNs obtained by PECNV exhibited good concordance with ASCAT. For example, the estimated ACNs attained by EXCAVATOR and ExomeCNV were 2.26 and 2.08 , respectively, in highly amplified tumor sample S1 (Table I), where the ACN was 3.62, predicted by ASCAT. The difference between EXCAVATOR, ExomeCNV and ASCAT was 1.36 and 1.54, respectively, whereas PECNV predicted a value of 3.60 for ACN, which was close to the ground truth. Additionally, the average absolute difference of ACN between the WES based methods and ASCAT were calculated for the 8 TNBC samples. The average absolute difference of ACN for EXCAVATOR and ExomeCNV were 0.49 and 0.47, respectively. Compared with EXCAVATOR and ExomeCNV, PECNV markedly improved the performance with an average absolute difference of 0.14 . These results 

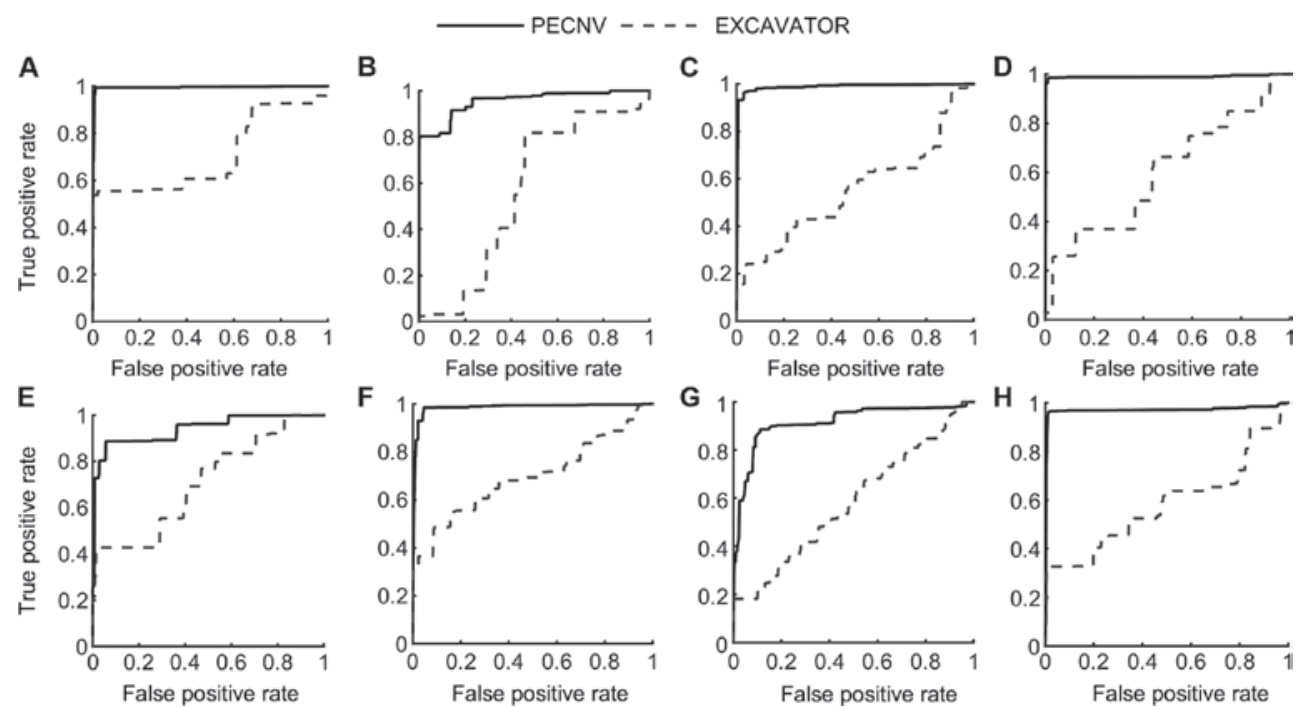

Figure 5. Receiver operating characteristic curves of PECNV and EXCAVATOR for detection of copy number variants in 8 real primary triple-negative breast cancer datasets. Samples (A) S1; (B) S2; (C) S3; (D) S4; (E) S5; (F) S6; (G) S7 and (H) S8.

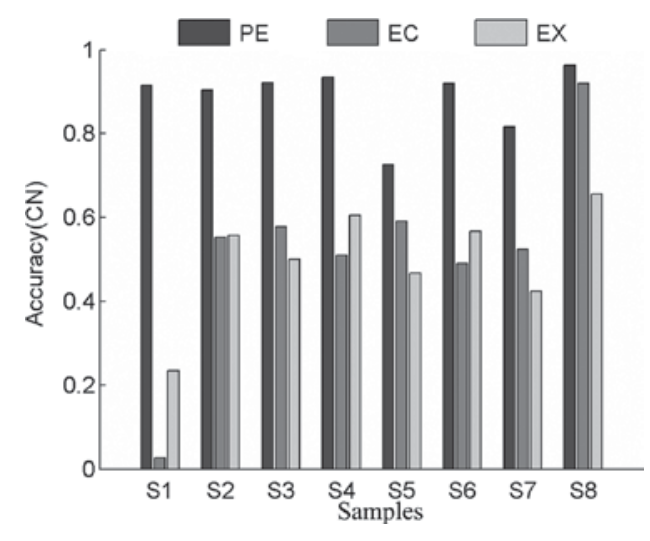

Figure 6. Accuracy of PECNV, ExomeCNV and EXCAVATOR in detecting copy number variants. CN, copy number; PE, PECNV; EX, EXCAVATOR; $\mathrm{EC}$, ExomeCNV.

suggested that PECNV has the potential for inferring tumor impurity and ACNs in complicated tumor samples.

Comparisons of $C N V$ detection in TNBC datasets. In order to evaluate the accuracy of PECNV, sensitivity and specificity of the three methods were determined (Fig. 4). The genomic aberration profiles of the 8 TNBC samples are summarized in Table II. The samples share the same number of exons, while the number of copy number gains or losses varied. Sample S1 contained the highest number of CNVs, while samples S4 had the lowest number of CNVs. In general, EXCAVATOR had the worst performance in terms of sensitivity, with an average value of 0.18 . Among all tumor samples, the highest sensitivity was obtained in tumor sample S1. Consistent with the finding in assessment of the simulated data, it was found that EXCAVATOR tends to be conservative in identifying CNVs. In comparison with EXCAVATOR, ExomeCNV achieved higher sensitivity in all samples. In particular, the highest sensitivity and specificity values were obtained in tumor sample S8 with ExomeCNV. However, ExomeCNV exhibited lower specificity in most tumor samples.
In general, ExomeCNV obtained better sensitivity and lower specificity compared with EXCAVATOR. By contrast, PECNV obtained the highest sensitivity across all samples accompanied by comparable specificity. Notably, PECNV obtained the best performance in tumor samples S2 and S4 in terms of sensitivity and specificity. Although for tumor samples S5 and S7, ExomeCNV exhibited better specificity, PECNV demonstrated better sensitivity for both samples. Taken together, the results demonstrated that PECNV exhibited a better overall performance in terms of sensitivity and specificity compared with EXCAVATOR and ExomeCNV, suggesting that PECNV has a good efficiency for detection of CNVs.

Apart from sensitivity and specificity, receiver operating characteristic (ROC) curves of the 8 samples (Fig. 5) were used to compare the performance of the different methods. In the present study, PECNV was compared to EXCAVATOR using ROC curves in the 8 TNBC samples. However, PECNV was not compared with ExomeCNV as ExomeCNV does not provide the possibility of calling $\mathrm{CNVs}$, which are required to generate the ROC curve. As shown in Fig. 5, the ROC curves of PECNV were above EXCAVATOR in all 8 samples, which indicate that PECNV has better performance compared with EXCAVATOR. Additionally, the area under the curve (AUC) was calculated to compare the results. The mean AUC of the 8 samples obtained by PECNV was 0.96 , while the value for EXCAVATOR was 0.62 , which suggested that PECNV has a higher detection power.

Comparison of copy number accuracy in real tumor datasets. In order to comprehensively evaluate the performance of different methods, values for copy number accuracy were also calculated for all tumor samples. As shown in Fig. 6, ExomeCNV exhibited higher accuracy compared with EXCAVATOR in 4 tumor samples, which indicates its power in detecting CNVs. PECNV achieved higher levels of accuracy in all tumor samples compared with ExomeCNV and EXCAVATOR, with average copy number accuracy values of $0.89,0.52$ and 0.50 for PECNV, ExomeCNV and EXCAVATOR, respectively. Overall, PECNV demonstrated better performance in accuracy in the detection of copy number in real WES datasets. 
Comparison of $\mathrm{LOH}$ detection in real tumors. In the present study, the performance of different methods for $\mathrm{LOH}$ detection in exonic regions was also evaluated. LOHs represent another type of somatic aberrations in the cancer genome (21). However, since only ExomeCNV is capable of detecting LOH using WES data $(16,30)$, the results of both PECNV and ExomeCNV obtained from all tumor samples were compared. As shown in Table III, the results of ExomeCNV suggested significant bias in $\mathrm{LOH}$ detection with a low specificity and a high sensitivity. For example, in tumor sample S1, the accuracy of ExomeCNV was 0.25 .

On the contrary, PECNV achieved balanced performance with satisfactory levels of sensitivity and specificity. Notably, the levels of accuracy in all 8 samples were markedly higher compared with ExomeCNV. In conclusion, PECNV was able to efficiently detect $\mathrm{LOH}$ regions in tumor WES data.

\section{Discussion}

In the present study, a novel method, PECNV, for accurate identification of copy number and LOH in tumor WES datasets was described. PECNV adopts a comprehensive correction and normalization procedure for eliminating batch effect and mapping bias confronted in exome sequencing. Additionally, PECNV is able to reduce the side effects of batch effect and mapping bias by automatically estimating and correcting tumor impurity and signal baseline shift, which enables an improved performance compared over other existing methods. Compared with EXCAVATOR and ExomeCNV, which dismisses BAF signals or take separate analysis of Log Ratio and BAF signals, PECNV simultaneously combines Log Ratio and BAF signals in modeling and parameters estimation, which results in an increased ability to detect $\mathrm{CNV}$ and $\mathrm{LOH}$.

However, the PECNV method has several limitations. Although PECNV is able to accurately infer tumor impurity up to $80 \%$, it may fail as tumor impurity continues to rise. In such case, both Log Ratio and BAF signals become extremely attenuated and amplification or deletion regions are hard to be distinguished from normal regions. As a result, PECNV may have difficulty in detecting somatic aberrations.

Another limitation is related to tumor heterogeneity. The underlying assumption adopted in PECNV is that only one type of aberration is allowed in each exonic region. In practice, the hypothesis may be rejected in the presence of heterogeneity. For example, Oesper et al (31) reported in tumor progression that multiple tumor subclones may appear in somatic cells. Therefore, detection of CNVs in heterogeneous samples is challenging. Currently, few methods have been developed to identify CNVs in heterogeneous tumor samples with WES sequencing. Sophisticated computational methods and in-depth biological analysis are required to address this issue $(28,32)$. PECNV and other previous studies may help towards solving this challenging task.

\section{Acknowledgements}

The manuscript was prepared using a limited access dataset obtained from the British Columbia Cancer Agency Branch (BCCA) and does not necessarily reflect the views or opinions of the BCCA. The present study was supported by the National
Natural Science Foundation of China (grant nos. 61571414, 61471331 and 31100955).

\section{References}

1. Albertson DG, Collins C, McCormick F and Gray JW: Chromosome aberrations in solid tumors. Nat Genet 34: 369-376, 2003.

2. Weir BA, Woo MS, Getz G, Perner S, Ding L, Beroukhim R, Lin WM, Province MA, Kraja A, Johnson LA, et al: Characterizing the cancer ge-nome in lung adenocarcinoma. Nature 450: 893-898, 2007.

3. Carén H, Kryh H, Nethander M, Sjöberg RM, Träger C, Nilsson S, Abrahamsson J, Kogner P and Martinsson T: High-risk neuroblastoma tumors with 11q-deletion display a poor prognostic, chromosome instabil-ity phenotype with later onset. Proc Natl Acad Sci USA 107: 4323-4328, 2010.

4. Solinas-Toldo S, Lampel S, Stilgenbauer S, Nickolenko J, Benner A, Döhner H, Cremer T and Lichter P: Matrix-based comparative genomic hybridization: Biochips to screen for genomic imbalances. Genes Chromosomes Cancer 20: 399-407, 1997.

5. Park PJ: Experimental design and data analysis for array comparative genomic hybridization. Cancer Invest 26: 923-928, 2008.

6. McCarroll SA, Kuruvilla FG, Korn JM, Cawley S, Nemesh J, Wysoker A, Shapero MH, de Bakker PI, Maller JB, Kirby A, et al: Integrated detection and population-genetic analysis of SNPs and copy number variation. Nat Genet 40: 1166-1174, 2008

7. Peiffer DA, Le JM, Steemers FJ, Chang W, Jenniges T, Garcia F, Haden K, Li J, Shaw CA, Belmont J, et al: High-resolution genomic profiling of chromosomal aberrations using Infinium whole-genome genotyping. Genome Res 16: 1136-1148, 2006.

8. Metzker ML: Sequencing technologies-the next generation. Nat Rev Genet 11: 31-46, 2010.

9. Morozova $\mathrm{O}$ and Marra MA: Applications of next-generation sequenc-ing technologies in functional genomics. Genomics 92: 255-264, 2008.

10. Wheeler DA, Srinivasan M, Egholm M, Shen Y, Chen L, McGuire A, He W, Chen YJ, Makhijani V, Roth GT, et al: The complete genome of an individual by massively parallel DNA sequencing. Nature 452: 872-876, 2008.

11. Teer JK and Mullikin JC: Exome sequencing: The sweet spot before whole genomes. Hum Mol Genet 19: R145-R151, 2010.

12. Clark MJ, Chen R, Lam HY, Karczewski KJ, Chen R, Euskirchen G, Butte AJ and Snyder M: Performance comparison of exome DNA sequencing technologies. Nat Biotechnol 29: 908-914, 2011.

13. Ng SB, Turner EH, Robertson PD, Flygare SD, Bigham AW Lee C, Shaffer T, Wong M, Bhattacharjee A, Eichler EE, et al: Targeted capture and massively parallel sequencing of 12 human exomes. Nature 461: 272-276, 2009.

14. Reese SE, Archer KJ, Therneau TM, Atkinson EJ, Vachon CM, de Andrade M, Kocher JP and Eckel-Passow JE: A new statistic for identifying batch effects in high-throughput genomic data that uses guided principal component analysis. Bioinformatics 29: 2877-2883, 2013.

15. Karakoc E, Alkan C, O'Roak BJ, Dennis MY, Vives L, Mark K, Rieder MJ, Nickerson DA and Eichler EE: Detection of structural variants and indels within exome data. Nat Methods 9: 176-178, 2012.

16. Sathirapongsasuti JF, Lee H, Horst BA, Brunner G, Cochran AJ, Binder S, Quackenbush J and Nelson SF: Exome sequencing-based copy-number variation and loss of heterozygosity detection: ExomeCNV. Bioinformatics 27: 2648-2654, 2011.

17. Krumm N, Sudman PH, Ko A, O'Roak BJ, Malig M, Coe BP; NHLBI Exome Sequencing Project, Quinlan AR, Nickerson DA and Eichler EE: Copy number variation detection and genotyping from exome sequence data. Genome Res 22: 1525-1532, 2012.

18. Fromer M, Moran JL, Chambert K, Banks E, Bergen SE, Ruderfer DM, Handsaker RE, McCarroll SA, O'Donovan MC, Owen MJ, et al: Discovery and statistical genotyping of copy-number variation from whole-exome sequencing depth. Am J Hum Genet 91: 597-607, 2012

19. Backenroth D, Homsy J, Murillo LR, Glessner J, Lin E, Brueckner M, Lifton R, Goldmuntz E, Chung WK and Shen Y: CANOES: Detecting rare copy number variants from whole exome sequencing data. Nucleic Acids Res 42: e97, 2014.

20. Magi A, Tattini L, Cifola I, D'Aurizio R, Benelli M, Mangano E, Battaglia C, Bonora E, Kurg A, Seri M, et al: EXCAVATOR: Detecting copy number variants from whole-exome sequencing data. Genome Biol 14: R120, 2013. 
21. Li A, Liu Z, Lezon-Geyda K, Sarkar S, Lannin D, Schulz V, Krop I, Winer E, Harris L and Tuck D: GPHMM: An integrated hidden Markov model for identification of copy number alteration and loss of heterozygosity in complex tumor samples using whole genome SNP arrays. Nucleic Acids Res 39: 4928-4941, 2011.

22. Li H, Handsaker B, Wysoker A, Fennell T, Ruan J, Homer N, Marth G, Abecasis G and Durbin R; 1000 Genome Project Data Processing Subgroup: The sequence alignment/map format and SAMtools. Bioinformatics 25: 2078-2079, 2009.

23. Yoon S, Xuan Z, Makarov V, Ye K and Sebat J: Sensitive and accurate detection of copy number variants using read depth of coverage. Genome Res 19: 1586-1592, 2009.

24. Mortazavi A, Williams BA, McCue K, Schaeffer L and Wold B: Mapping and quantifying mammalian transcriptomes by RNA-Seq. Nat Methods 5: 621-628, 2008.

25. Bilmes JA: A gentle tutorial of the EM algorithm and its-application to parameter estimation for Gaussian mixture and hidden Markov models. Int Comput Sci Inst 4: 126, 1998.

26. Olshen AB, Venkatraman ES, Lucito R and Wigler M: Circular binary segmentation for the analysis of array-based DNA copy number data. Biostatistics 5: 557-572, 2004.
27. Møller MF: A scaled conjugate gradient algorithm for fast supervised learning. Neural Net 6: 525-533, 1993.

28. Yu Z, Liu Y, Shen Y, Wang M and Li A: CLImAT: Accurate detection of copy number alteration and loss of heterozygosity in impure and aneuploid tumor samples using whole-genome sequencing data. Bioinformatics 30: 2576-2583, 2014.

29. Shah SP, Roth A, Goya R, Oloumi A, Ha G, Zhao Y, Turashvili G, Ding J, Tse K, Haffari G, et al: The clonal and mutational evolution spectrum of primary triple-negative breast cancers. Nature 486: 395-399, 2012

30. Van Loo P, Nordgard SH, Lingjærde OC, Russnes HG, Rye IH, Sun W, Weigman VJ, Marynen P, Zetterberg A, Naume B, et al: Allele-specific copy number analysis of tumors. Proc Natl Acad Sci USA 107: 16910-16915, 2010.

31. Oesper L, Mahmoody A and Raphael BJ: THetA: Inferring intra-tumor heterogeneity from high-throughput DNA sequencing data. Genome Biol 14: R80, 2013.

32. Tan R, Wang Y, Kleinstein SE, Liu Y, Zhu X, Guo H, Jiang Q, Allen AS and Zhu M: An evaluation of copy number variation detection tools from whole-exome sequencing data. Hum Mutat 35: 899-907, 2014. 